SUBJECT AREAS:

HAEMATOLOGICAL

DISEASES

BIOMARKER RESEARCH

EYE MANIFESTATIONS

DIAGNOSTIC MARKERS

Received

21 May 2013

Accepted

1 October 2013

Published

5 December 2013

Correspondence and requests for materials should be addressed to Y.O. (yoko@z7.keio.

\section{Graft-vs-Host-Disease (GVHD) Consensus Group: Proposed Diagnostic Criteria for Chronic GVHD (Part I)}

Yoko Ogawa', Stella K. Kim², Reza Dana ${ }^{3}$, Janine Clayton ${ }^{4}$, Sandeep Jain ${ }^{5}$, Mark I. Rosenblatt ${ }^{6}$, Victor L. Perez ${ }^{7}$, Hasanain Shikari ${ }^{3}$, Anjo Riemens ${ }^{8} \&$ Kazuo Tsubota ${ }^{1}$

${ }^{1}$ Department of Ophthalmology, Keio University School of Medicine, Tokyo, Japan, ${ }^{2}$ MD Anderson Cancer Center, Houston, Texas, USA, ${ }^{3}$ Massachusetts Eye and Ear Infirmary, Department of Ophthalmology, Harvard Medical School, Boston, Massachusetts, USA, ${ }^{4}$ National Institute of Health, Bethesda, Maryland, USA, ${ }^{5}$ Corneal Neurobiology Laboratory, University of Illinois at Chicago, Chicago, Illinois, USA, ${ }^{6}$ Weill Cornell Medical College, New York, New York, USA, ${ }^{7}$ Bascom Palmer Eye Institute, University of Miami Miller School of Medicine, Miami, Florida, USA, ${ }^{8}$ University Medical Center Utrecht, Utrecht, the Netherlands.

The International Chronic Ocular GVHD Consensus Group held 4 working meetings to define new diagnostic metrics for chronic ocular graft-versus-host disease (GVHD). After considering the factors currently used to diagnose chronic ocular GVHD, the Consensus Group identified 4 subjective and objective variables to measure in patients following allogeneic hematopoietic stem cell transplantation (HSCT): OSDI, Schirmer's score without anesthesia, corneal staining, and conjunctival injection. Each variable was scored 0-2 or 0-3, with a maximum composite score of 11 . Consideration was also given to the presence or the absence of systemic GVHD. On the basis of their composite score and the presence or absence of systemic GVHD, patients were assigned to one of three diagnostic categories: NO, PROBABLE, or DEFINITE ocular GVHD. New diagnostic criteria for chronic ocular GVHD are presented by the Consensus Group. Validation studies are needed to identify the best combination of the proposed metrics to maximize diagnostic sensitivity and specificity.

ry eye disease (DED) is one of the most common manifestations of chronic graft-versus-host disease (GVHD) and has been recognized as an important complication after allogeneic hematopoietic stem cell transplantation (HSCT) ${ }^{1-12}$. Since visual function and ocular symptoms are largely related to patient's quality of life ${ }^{13}$, ocular complications have a great impact on morbidity after successful HSCT. More than 25000 HSCT procedures are performed annually and the number is increasing worldwide ${ }^{14}$.

Historically, cGVHD was classified as limited or extensive based on the clinical findings in a small cohort of patients ${ }^{1}$. Recently, diagnostic criteria for cGVHD have been proposed by the National Institutes of Health $(\mathrm{NIH})^{15}$. As per the NIH consensus criteria definition, "diagnosis of cGVHD requires the presence of at least 1 diagnostic clinical sign of cGVHD (e.g., polikiloderma or esophageal web) or the presence of at least 1 distinctive manifestation (e.g., keratoconjunctivitis sicca) confirmed by pertinent biopsy or other relevant tests (e.g., Schirmer test) in the same or another organ." The NIH Consensus therefore notes that dry eye is a distinctive sign seen in cGVHD but insufficient in itself to establish a diagnosis of chronic GVHD. However, this precludes the early diagnosis of systemic GVHD in the presence of new onset DED after HSCT. In this paper, we compile recently emerging evidence that supports the classification of DED as a diagnostic clinical entity for chronic GVHD.

The purpose of international workshops on chronic ocular GVHD were:

1) To provide a consensus overview of chronic ocular GVHD.

2) To refine the definition and classification of chronic ocular GVHD.

3) To assess methods of diagnosis, evaluation, and grading of chronic ocular GVHD by reviewing previously reported literature.

4) To generate a proposal to change dry eye syndrome as a sufficient clinical entity for the diagnosis of cGVHD, address the diagnostic criteria, discuss the severity scores, and recommend an amendment to the NIH Consensus diagnostic criteria for the stem cell transplantation community. 
First, we would like to establish the consensus diagnostic criteria and classification for chronic ocular GVHD. Next, after validating a multicenter and prospective study using these criteria, we would like to propose the new diagnostic criteria for chronic GVHD based on ocular GVHD assessment. Our ultimate goal is to add ocular GVHD as a diagnostic sign for chronic GVHD.

\section{Results}

DED as a diagnostic sign of chronic GVHD. DED is commonly seen in patients with chronic GVHD $^{6,9,16-19}$, and may be considered a hallmark of chronic GVHD ${ }^{10,11,20-28}$. The proposed criteria for diagnosis and scoring of chronic systemic as well as ocular GVHD need to be validated in prospective studies ${ }^{15,29}$. A prospective evaluation of patients undergoing HSCT between 1995 and 1998, pre- and post-transplantation showed that dry eye was observed with greater frequency in patients with systemic chronic GVHD $(70.4 \%)$, than in patients without $(17.7 \% \text {; } \mathrm{P}<.005)^{3}$. Using Schirmer score for staging of ocular GVHD as proposed by the $\mathrm{NIH}$ consensus criteria, the false positive rate of diagnosis of ocular GVHD in patients with systemic GVHD was $19.4 \%$ and false negative rate was $22.7 \%$. Jacobs et al. have reported similar results ${ }^{10}$. In terms of temporal association of onset of ocular and systemic GVHD, Balaram et al. ${ }^{4}$ have reported ocular involvement in $62 \%$ patients with chronic GVHD. Twenty two percent of newly onset dry eye patients after HSCT presented with severe dry eye and conjunctival inflammation without features of systemic GVHD. They have also reported ocular involvement as a predecessor of systemic chronic $\mathrm{GVHD}^{3,4}$. Early diagnosis of chronic ocular GVHD enables adequate therapeutic intervention for ocular and systemic signs and symptoms.

There are several risk factors that predispose to the development of chronic GVHD. These include an episode of acute GVHD, age of donor or recipient, and donor-recipient gender disparity ${ }^{5}$. Female donor to male recipient transplants were shown to result in an increased ratio of severe/total dry eye than in male donor to female recipient ${ }^{30}$. It is well recognized that the stem cell source can impact GVHD risk ${ }^{31}$, and Uchino, M., et al. reported increased frequency of chronic ocular GVHD post peripheral blood stem cell transplantation (PBSCT) compared to bone marrow (BMT) or cord blood transplantation $(\mathrm{CBT})^{32}$. They demonstrated the relationship between the development of systemic GVHD and dry eye after HSCT. Patients with GVHD had a higher Odds Ratio for dry eye post-BMT (OR $12.28,95 \%$ CI 2.48 to 60.5 ) and post-CBT (OR $13.8,95 \%$ CI 0.4 to 448.6 ) compared to post-PBSCT (OR 3, 95\% CI 0.4 to 22.7). These findings suggest that dry eye in patients post-BMT/CBT was a predictor for occurrence of systemic GVHD. In PBSCT, dry eye may be masked by intensive systemic immunosuppression. It was also noted that late onset severe dry eye can precede non-ocular systemic GVHD. This leads us to suggest that dry eye is an important sign for diagnosing or treating systemic chronic GVHD.

GVHD has the potential to affect all mucosal surfaces, including ocular, oral, vaginal, and gastrointestinal areas ${ }^{15}$. Ocular surface mucosa is a representative target organ of chronic GVHD

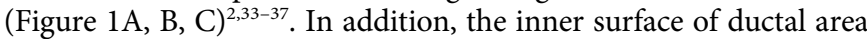
is composed of mucosal membrane which is frequently targeted $\mathrm{T}$ cells or other inflammatory cells (Figure 2A), ductal area is significantly affected in target organs such as lacrimal gland, meibomian (Figure 2A, B) gland, nasolacrimal ducts and salivary gland, as well as hepatic bile ducts, and lung ducts ${ }^{2,38-42}$.

Conjunctival involvement in chronic GVHD has been reported as a sign of severe chronic GVHD' ${ }^{2}$ Kaplan-Meier analysis of survival demonstrated a decreased survival of patients with GVHD presenting with conjunctival involvement ${ }^{2}$. It has been proposed by Jabs et al that the conjunctiva may mirror systemic mucosal membrane such as intestine, lung, and oral mucosa. They described that conjunctival involvement by GVHD may represent "a distinct clinical finding"

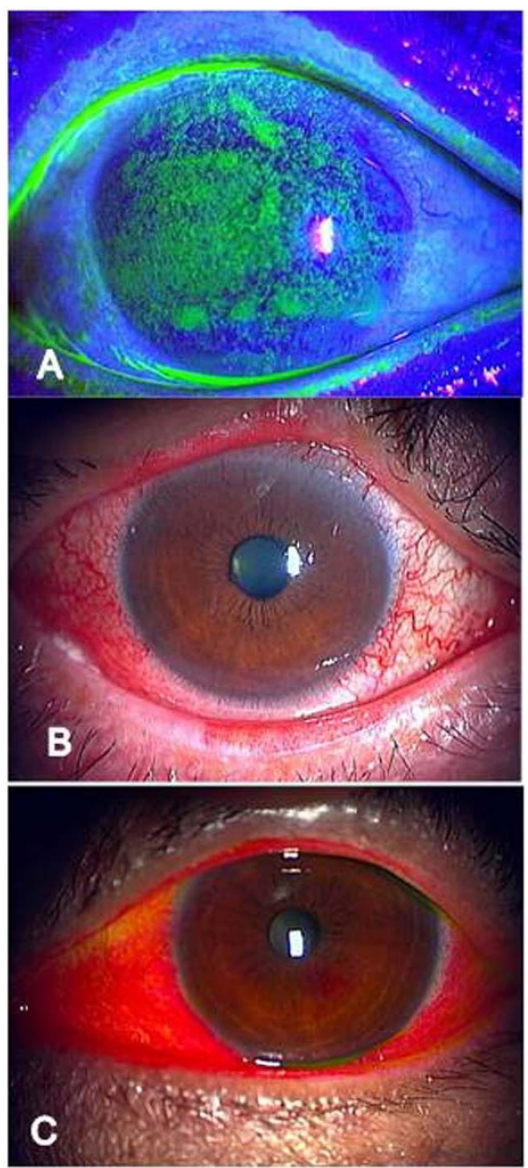

Figure $1 \mid$ Typical ocular surface findings of chronic ocular GVHD. (A) A representative biomicroscopic image of fluorescein staining (Severity score 3) is shown. Note diffuse punctate epitheliopathy on the cornea of a patient with chronic GVHD related dry eye. Gray lines (Meibolines on upper and lower lid margin) are distorted. (B) Note severe conjunctival injection on damaged ocular surface. (Severity score 2. (C) Note Rose Bengal staining of conjunctiva on severely damaged ocular surface.

The description might leads to dry eye (keratoconjunctivitis sicca) being recognized as a "distinct sign" in chronic GVHD, particularly in reference to the NIH consensus criteria.

Conjunctivitis may present concurrently or precede the onset of dry eye in chronic GVHD. Conjunctival inflammation may be a primary manifestation of chronic GVHD or secondary to severe dry eye. The role of conjunctival biopsy for ocular GVHD remains unclear. A recent report studied conjunctival biopsy specimen in symptomatic patients suspected of ocular $\mathrm{GVHD}^{34}$. Using a modified Lerner classification designed for skin GVHD histopathology, AuwHaedrich and colleagues found that the modified grade III-IV bulbar conjunctival inflammation cohort compared to modified grade I-II showed shorter survival in Kaplan-Meier analysis. This study supports the earlier observation made by Jabs et al that conjunctival involvement may confer a prognostic value.

We can detect the ocular surface change of chronic GVHD directly by using a biomicroscope and comprehensive variables for diagnosing dry eye disease after HSCT. Therefore, early detection, precise diagnosis, and treatment of dry eye in ocular GVHD as well as systemic GVHD are necessary to prevent the blindness for long term survivors.

Based on these reports, dry eye post-HSCT has been established as a significant finding in the spectrum of systemic involvement in chronic GVHD. Since dry eye can precede clinical signs in other organs, we propose the inclusion of dry eye as a concrete diagnostic 

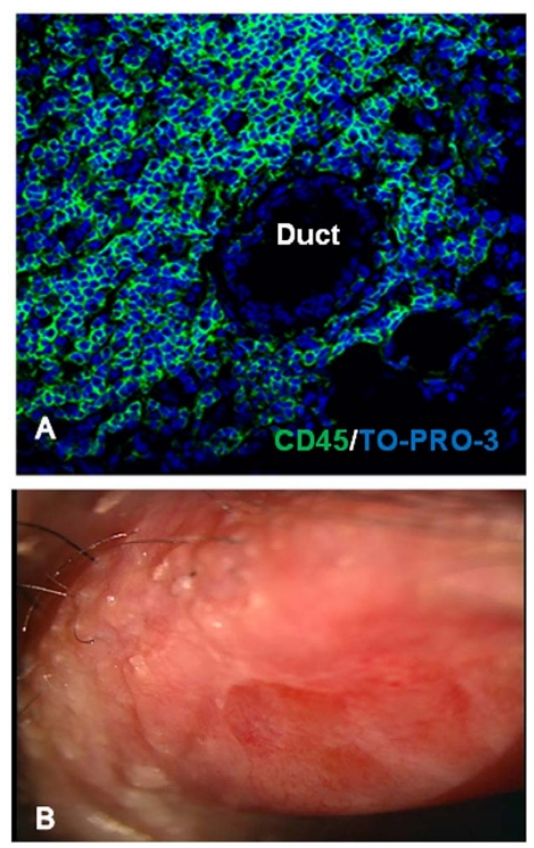

Figure $2 \mid$ Histological findings of lacrimal gland ducts and clinical findings of orifices of meibomian gland and Zeiss gland on eye lid margin. (A) Typical histological findings of lacrimal gland focusing on medium sized duct and from a patient of chronic GVHD for diagnostic purpose and clinical findings around ductal orfices of meibomian and Zeiss gland in the lid margin of a 21-year-old male patient with chronic GVHD. CD45 immunostaining of inflammatory cells in lacrimal gland from a chronic GVHD patient. An abundant $\mathrm{CD} 45^{+}$inflammatory cell infiltration at periductal areas of lacrimal gland chronic GVHD. A 39-yearold female patient at 2 months after onset of cGVHD related dry eye. Original magnification: X630. (B) Note total obstruction of meibomian gland and Zeis gland orifices and severely fibrotic tarsal conjunctiva. Upper eye lid, right eye. A 21-year-old male suffering from chronic GVHD.

sign of chronic GVHD without being substantiated by involvement in other organ systems as originally recommended by the NIH working committee.

Proposed diagnostic criteria and severity of grading system. A collaborative, prospective-multicenter study from the USA, the Netherlands, and Japan on ocular GVHD has been proposed. Based on discussion and consensus at the 1st Chronic Ocular GVHD meeting, the parameters for diagnosis include 1) Ocular Surface Disease Index (OSDI $\left.)^{43,44}, 2\right)$ Schirmer's test score without anesthesia ${ }^{45}$, 3) Corneal fluorescein staining (Figure 3) ${ }^{45,46}$, and 4) conjunctival injection (Figure 4$)^{47}$. Ocular GVHD classification will be based on the baseline examination as threshold scores for grading. The proposed grading system is shown in Tables 1 and 2 . Severity scores $0,1,2$, and 3 will be assigned to OSDI, corneal fluoresecein staining, and Schirmer's score ${ }^{4-46}$. Conjunctival injection will be scored 0,1 , and 2 (Table 1$)^{47}$. The corneal fluorescein staining score ranged from 0 to 3 points (Grade $0=$ no staining, Grade $1=$ minimal staining, Grade $2=$ mild $/$ moderate staining, Grade $3=$ severe staining) (Figure 3$)^{46}$. The conjunctival hyperemia score of the conjunctiva ranged from 0 to 2 points (Grade $0=$ none, Grade $1=$ mild/moderate, Grade $2=$ severe) (Figure 4$)^{47}$. Any score above 1 points is regarded as abnormal.

Disease severity will be graded as none, mild/moderate and severe based on an aggregate of scores for each parameter (Table 1). Based on the presence or absence of systemic GVHD and the aggregate scores assessed, a diagnosis of ocular GVHD will be made (Table 2). In the presence of systemic GVHD, score $0-3$ indicates absence of

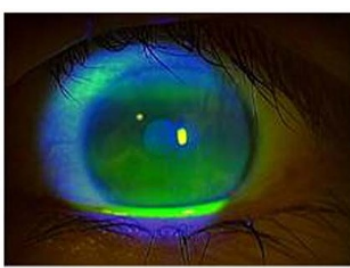

\section{Grade 0}

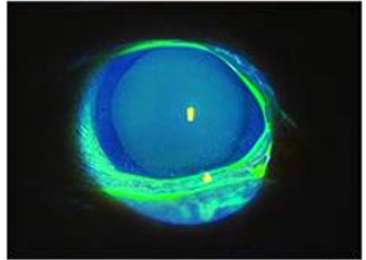

Grade 1

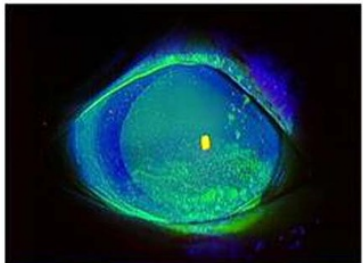

Grade 2

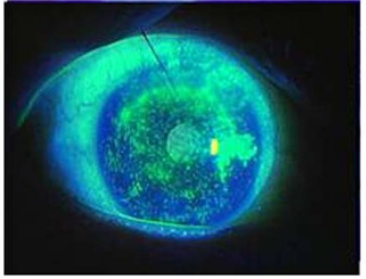

\section{Grade 3}

Figure 3 | Slitlamp micrograph of grading scale for corneal fluorescein staining. Grading scale of corneal fluorescein staining score ranged from 0 to 3 points (Grade $0=$ no staining, Grade $1=$ minimal staining, Grade $2=$ mild/moderate staining, Grade 3 = severe staining).

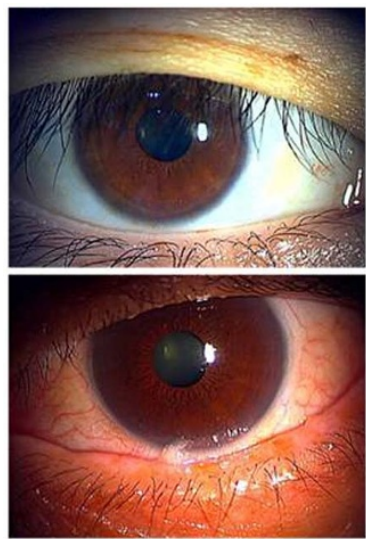

\section{Grade 0}

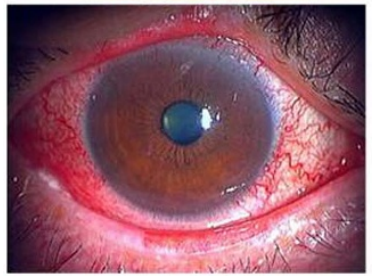

Grade 1

\section{Grade 2}

Figure 4 Slit lamp micrograph of grading scale for conjunctival injection. Grading scale of conjunctival injection score ranged from 0 to 2 points $($ Grade $0=$ none, Grade $1=$ mild/moderate injection, Grade $2=$ severe injection). 


\begin{tabular}{lrrrl} 
Table 1 | Severity scale in chronic ocular GVHD & \\
$\begin{array}{l}\text { Severity scores } \\
\text { (points) }\end{array}$ & $\begin{array}{r}\text { Schirmer's } \\
\text { test (mm) }\end{array}$ & CFS (points) & OSDI (points) & Coni (points) \\
\hline 0 & $>15$ & 0 & $<13$ & None \\
1 & $11-15$ & $<2$ & $13-22$ & Mild/Moderate \\
2 & $6-10$ & $2-3$ & $23-32$ & Severe \\
3 & $\leq 5$ & $\geq 4$ & $\geq 33$ & \\
\hline
\end{tabular}

CFS; corneal fluorescein staining, OSDI; Ocular Surface Disease Index. Conj; conjunctival injection. Severity classification; Total score (points); (Schirmer's test score+ CFS score+ OSDI score + Conj injection score) $=$ None;0-4, Mild/Moderate; $5-8$, Severe, 9-1 1.

ocular GVHD; score 4-5 indicates "probable" ocular GVHD and score $\geq 6$ indicates "definite" ocular GVHD. In the absence of systemic GVHD, score 0-5 indicates absence of ocular GVHD, score 67 indicates "probable" ocular GVHD and score $\geq 8$ indicates "definite" ocular GVHD (Table 2). Based on the above criteria, data collected at each center will be analyzed and validated at a central location and a proposal to amend the NIH consensus criteria will be presented.

\section{Discussion}

DED is responsible for a significant decrease in the quality of life in allogeneic transplant recipients. DED is currently listed as a distinctive sign, and not a diagnostic criteria sufficient alone for establishing a diagnosis of chronic GVHD by the NIH consensus criteria ${ }^{15}$.

The NIH consensus criteria diagnosis and staging working group report defined ocular criteria for diagnosis as "new ocular sicca documented by low Schirmer test values with a mean value of both eyes $\leq 5 \mathrm{~mm}$ at 5 minutes or a new onset of keratoconjunctivitis sicca by slit-lamp examination with mean values of 6 to $10 \mathrm{~mm}$ on the Schirmer test accompanied by distinctive manifestations in at least 1 other organ"15. While recognizing that the NIH criteria were developed for the use of transplant physicians in prospective clinical trials for chronic GVHD, the ocular criteria need to be evaluated by ophthalmologists. Decreased Schirmer's score, signs, and symptoms of DED must be assessed simultaneously in correlation with the clinical findings, since infection, trauma, and side effects from prolonged use of topical medications may also present with ocular surface disease. Using the Schirmer's score as a sole defining criterion for the diagnosis of chronic ocular GVHD would be inadequate. The high false positive and negative rates of diagnosis of ocular GVHD if based solely on the Schirmer score have been reported by various groups ${ }^{10}$. In addition, the importance of a comprehensive ocular evaluation has been stressed. It is commonly accepted that $5 \mathrm{~mm}$ of wetting denotes tear deficiency when the test is performed without anesthesia. However, a comparable diagnostic cut-off value for Schirmer's test with anesthesia is not as clear. Other limitations include the errors in measurement induced by reflex tearing and the influence of external environmental factors brings its infallibility into question ${ }^{48}$. Patient symptom scores, ocular surface staining, tear film dynamics, are all important in assessing a patient with dry eye in ocular GVHD, especially in assessing systemic chronic GVHD. Diagnostic procedures for dry eye have been reported by the International Dry Eye Workshop (DEWS) report ${ }^{13}$. Modification

\begin{tabular}{|lccc|}
\hline Table 2 | Diagnosis of chronic ocular GVHD & \\
& $\begin{array}{c}\text { None } \\
\text { (points) }\end{array}$ & $\begin{array}{c}\text { Probable GVHD } \\
\text { (points) }\end{array}$ & $\begin{array}{c}\text { Definite GVHD } \\
\text { (points) }\end{array}$ \\
\hline Systemic GVHD(-) & $0-5$ & $6-7$ & $\geq 8$ \\
Systemic GVHD(+) & $0-3$ & $4-5$ & $\geq 6$ \\
\hline
\end{tabular}

of the proposed criteria may allow for standardization of dry eye assessment in chronic GVHD because patient evaluation, followup and treatment regimens differ at various centers. Irrespective, a careful examination by an ophthalmologist perceptive to the diagnosis of ocular GVHD serves a crucial role. A collaborative dialogue between the ophthalmologists and BMT specialists can lead to improved management of chronic GVHD in general ${ }^{3,6}$.

Considering conjunctival staining as parameter to assess ocular GVHD, there is no reliable, standardized, and readily adopted way to assess the conjunctival staining element of ocular GVHD at present time. Conjunctival staining is more variable even than corneal staining. In addition, while there are patients with conjunctival staining that don't stain corneas, the diagnostic criteria are ill-defined. Therefore, we exclude the conjunctival staining as the consensus parameter for the diagnosis of chronic ocular GVHD at present time. The NIH proposed scoring system for ocular GVHD (Table 3$)^{15}$ is easy to understand and can be performed without a clinical examination even by a non-ophthalmologist. However, it may not be able to accurately assess the severity of the disease. There is an inherent limitation in a scale that relies on behavior rather than objective findings.

The current grading systems available for ocular GVHD rely on clinical findings only. Jabs' proposed stages of conjunctival ocular GVHD are applicable to acute $\mathrm{GVHD}^{2}$; Robinson et al proposed a grading system for chronic ocular GVHD. Both are based on clinical findings ${ }^{36}$. Baseline profiles of ocular surface and tear film dynamics, in patients post-HSCT with or without chronic GVHD related dry eye have been reported. In this report, baseline values indicative of dry eye, using modern diagnostic techniques were demonstrated ${ }^{49}$. These data may be useful for an additional detailed evaluation of chronic ocular GVHD. Analysis of the efficacy of topical and systemic treatment for chronic ocular GVHD must take into account the baseline clinical findings and their severity ${ }^{49}$. It is our opinion, that a metric inclusive of patient symptom evaluation by the ocular surface disease index (OSDI) could be used to evaluate the effect on activities of daily living rather than the frequency of instillation of eye drops.

The recommendation of the current Chronic Ocular GVHD Workshop is to propose changes in the NIH Consensus Diagnostic Criteria to include chronic ocular GVHD, as a diagnostic criteria sufficient alone to establish a diagnosis of chronic GVHD. We also propose and amendment of the severity scoring criteria for chronic ocular GVHD by validating the metric proposed by our group. This will be done using the data generated at the centers enrolled in this study. Once validated, chronic ocular GVHD-specific metric, may be a valuable tool for future clinical trials and may help patients with ocular and systemic chronic GVHD. Early prophylaxis, diagnosis and treatment can be accomplished by examining the eye in detail.

Table 3 | Eye scoring of chronic GVHD by NIH consensus development project ${ }^{15}$

\begin{tabular}{lllll} 
Eyes & Score 0 & Score 1 & Score 2 & Score 3 \\
\hline Mean tear test $(\mathrm{mm}):$ & No & Mild dry eye symptoms not & Moderate dry eye & Severe dry eye symptoms significantly \\
$>10$ & symptoms & affecting ADL (requiring & symptoms partially affecting & affecting ADL (special eyeware to relieve \\
$6-10$ & & eyedrops OR asymptomatic & ADL (requiring drops $>$ & pain) OR unable to work because of \\
$<5$ & & signs of keratoconjunctivitis & $3 \times$ per day or punctal plugs), & ocular symptoms OR loss of vision caused \\
Not done & sicca & WITHOUT vision impairment & by keratoconjunctivitis sicca \\
\hline
\end{tabular}


In this regard, a prospective study evaluating a comprehensive ophthalmic evaluation pre- and post-transplantation to diagnose new onset dry eye or other chronic ocular GVHD-related and nonGVHD complications is needed. It is necessary to communicate in detail concerning the dose of systemic and local immunosuppressant and the time of commencement and cessation for treatment between transplant internists and ophthalmologists.

\section{Methods}

The International Ophthalmology Consensus Conference on Chronic Ocular GVHD was held comprising nine ophthalmologists from the USA, the Netherlands and Japan. The workshop occurred with the purpose of reviewing the published data, gathering a consensus from those in the field, with clinical practice and research interests in chronic ocular GVHD, and providing guidelines for diagnostic criteria

Four working meetings by the International Chronic Ocular GVHD Consensus Group were held. Each investigator participated in reviewing the data and the collective suggestions for the significance of DED in the diagnosis for systemic GVHD. The metric for diagnostic criteria, the severity of DED and relevance to systemic GVHD were discussed to identify currently used diagnostic variables in patients history and examination. Subjective and objective factors were considered and scores were assigned to each variable to reflect severity of the disease. Consideration was given to the presence or absence of systemic GVHD.

Lacrimal gland histology. Lacrimal gland biopsy specimens that had been obtained for diagnostic purposes were used for histological analysis.

This study was approved by the Keio University Institutional Review Boards (\#2009-0277). Written informed consent was obtained in advance from the patient in accordance with the principles expressed in the Declaration of Helsinki for human subjects.

1. Shulman, H. M. et al. Chronic graft-versus-host syndrome in man. A long-term clinicopathologic study of 20 Seattle patients. Am J Med 69, 204-217 (1980).

2. Jabs, D. A. et al. The eye in bone marrow transplantation. III. Conjunctival graftvs-host disease. Arch Ophthalmol 107, 1343-1348 (1989).

3. Ogawa, Y. et al. Dry eye after haematopoietic stem cell transplantation. $\mathrm{Br} J$ Ophthalmol 83, 1125-1130 (1999).

4. Balaram, M. \& Dana, M. R. Phacoemulsification in patients after allogeneic bone marrow transplantation. Ophthalmology 108, 1682-1687 (2001).

5. Couriel, D. R. et al. Extracorporeal photochemotherapy for the treatment of steroid-resistant chronic GVHD. Blood 107, 3074-3080 (2006).

6. Kim, S. K. Update on ocular graft versus host disease. Curr Opin Ophthalmol 17, 344-348 (2006).

7. Lew, J. \& Smith, J. A. Mucosal graft-vs-host disease. Oral Dis 13, 519-529 (2007)

8. Riemens, A., te Boome, L., Imhof, S., Kuball, J. \& Rothova, A. Current insights into ocular graft-versus-host disease. Curr Opin Ophthalmol 21, 485-494 (2010).

9. Townley, J. R., Dana, R. \& Jacobs, D. S. Keratoconjunctivitis sicca manifestations in ocular graft versus host disease: pathogenesis, presentation, prevention, and treatment. Semin Ophthalmol 26, 251-260 (2011).

10. Jacobs, R. et al. Prevalence and risk factors associated with development of ocular GVHD defined by NIH consensus criteria. Bone Marrow Transplant 47, 1470-1473 (2012).

11. Hessen, M. \& Akpek, E. K. Ocular graft-versus-host disease. Curr Opin Allergy Clin Immunol 12, 540-547 (2012).

12. Shikari, H., Antin, J. H. \& Dana, R. Ocular Graft-versus-Host Disease: A Review. Surv Ophthalmol (2013).

13. The definition and classification of dry eye disease: report of the Definition and Classification Subcommittee of the International Dry Eye WorkShop (2007). Ocul Surf 5, 75-92 (2007)

14. Ferrara, J. L., Levine, J. E., Reddy, P. \& Holler, E. Graft-versus-host disease. Lancet 373, 1550-1561 (2009).

15. Filipovich, A. H. et al. National Institutes of Health consensus development project on criteria for clinical trials in chronic graft-versus-host disease: I. Diagnosis and staging working group report. Biol Blood Marrow Transplant 11 , 945-956 (2005).

16. Dietrich-Ntoukas, T. et al. Diagnosis and treatment of ocular chronic graftversus-host disease: report from the German-Austrian-Swiss Consensus Conference on Clinical Practice in chronic GVHD. Cornea 31, 299-310 (2012).

17. Dignan, F. L. et al. Organ-specific management and supportive care in chronic graft-versus-host disease. Br J Haematol 158, 62-78 (2012).

18. Westeneng, A. C. et al. Ocular graft-versus-host disease after allogeneic stem cell transplantation. Cornea 29, 758-763 (2010).

19. Ogawa, Y., Shimmura, S., Dogru, M. \& Tsubota, K. Immune processes and pathogenic fibrosis in ocular chronic graft-versus-host disease and clinical manifestations after allogeneic hematopoietic stem cell transplantation. Cornea 29, S68-S77 (2010).

20. Riemens, A., Stoyanova, E., Rothova, A. \& Kuiper, J. Cytokines in tear fluid of patients with ocular graft-versus-host disease after allogeneic stem cell transplantation. Mol Vis 18, 797-802 (2012).
21. Inamoto, Y. et al. Validation of measurement scales in ocular graft-versus-host disease. Ophthalmology 119, 487-493 (2012).

22. Wang, Y. et al. Baseline profiles of ocular surface and tear dynamics after allogeneic hematopoietic stem cell transplantation in patients with or without chronic GVHD-related dry eye. Bone Marrow Transplant (2009).

23. Mencucci, R. et al. Ophthalmological aspects in allogenic bone marrow transplantation: Sjogren-like syndrome in graft-versus-host disease. Eur J Ophthalmol 7, 13-18 (1997).

24. Tichelli, A. Late ocular complications after bone marrow transplantation. Nouv Rev Fr Hematol 36 Suppl 1, S79-82 (1994).

25. Jack, M. K., Jack, G. M., Sale, G. E., Shulman, H. M. \& Sullivan, K. M. Ocular manifestations of graft-v-host disease. Arch Ophthalmol 101, 1080-1084 (1983).

26. Jabs, D. A. et al. The eye in bone marrow transplantation. II. Histopathology. Arch Ophthalmol 101, 585-590 (1983).

27. Hirst, L. W., Jabs, D. A., Tutschka, P. J., Green, W. R. \& Santos, G. W. The eye in bone marrow transplantation. I. Clinical study. Arch Ophthalmol 101, 580-584 (1983).

28. Franklin, R. M. et al. Ocular manifestations of graft-vs-host disease. Ophthalmology 90, 4-13 (1983).

29. Greinix, H. T. et al. Diagnosis and staging of chronic graft-versus-host disease in the clinical practice. Biol Blood Marrow Transplant 17, 167-175 (2011).

30. Kamoi, M. et al. Donor-recipient gender difference affects severity of dry eye after hematopoietic stem cell transplantation. Eye (Lond) 25, 860-865 (2011).

31. Takahashi, S. et al. Comparative single-institute analysis of cord blood transplantation from unrelated donors with bone marrow or peripheral blood stem-cell transplants from related donors in adult patients with hematologic malignancies after myeloablative conditioning regimen. Blood 109, 1322-1330 (2007).

32. Uchino, M. et al. Comparison of stem cell sources in the severity of dry eye after allogeneic haematopoietic stem cell transplantation. Br J Ophthalmol 96, 34-37 (2012).

33. Rojas, B. et al. Cell populations and adhesion molecules expression in conjunctiva before and after bone marrow transplantation. Exp Eye Res 81, 313-325 (2005).

34. Auw-Haedrich, C. et al. Histological and immunohistochemical characterisation of conjunctival graft vs host disease following haematopoietic stem cell transplantation. Graefes Arch Clin Exp Ophthalmol 245, 1001-1007 (2007).

35. Ogawa, Y. et al. Epithelial mesenchymal transition in human ocular chronic graftversus-host disease. Am J Pathol 175, 2372-2381 (2009).

36. Robinson, M. R. et al. Topical corticosteroid therapy for cicatricial conjunctivitis associated with chronic graft-versus-host disease. Bone Marrow Transplant 33, 1031-1035 (2004).

37. Tatematsu, Y. et al. Mucosal microvilli in dry eye patients with chronic GVHD. Bone Marrow Transplant 47, 416-425 (2012).

38. Hanada, R. \& Ueoka, Y. Obstruction of nasolacrimal ducts closely related to graftversus-host disease after bone marrow transplantation. Bone Marrow Transplant 4, 125-126 (1989).

39. Ogawa, Y. et al. A significant role of stromal fibroblasts in rapidly progressive dry eye in patients with chronic GVHD. Invest Ophthalmol Vis Sci 42, 111-119 (2001).

40. Kamoi, M. et al. Spontaneous lacrimal punctal occlusion associated with ocular chronic graft-versus-host disease. Curr Eye Res 32, 837-842 (2007).

41. Ban, Y. et al. Morphologic evaluation of meibomian glands in chronic graftversus-host disease using in vivo laser confocal microscopy. Mol Vis 17, 2533-2543 (2011).

42. Espana, E. M., Shah, S., Santhiago, M. R. \& Singh, A. D. Graft versus host disease: clinical evaluation, diagnosis and management. Graefes Arch Clin Exp Ophthalmol (2013).

43. Schiffman, R. M., Christianson, M. D., Jacobsen, G., Hirsch, J. D. \& Reis, B. L. Reliability and validity of the Ocular Surface Disease Index. Arch Ophthalmol 118, 615-621 (2000).

44. Miller, K. L. et al. Minimal clinically important difference for the ocular surface disease index. Arch Ophthalmol 128, 94-101 (2010).

45. Sullivan, B. D. et al. An objective approach to dry eye disease severity. Invest Ophthalmol Vis Sci 51, 6125-6130 (2010).

46. Bron, A. J., Evans, V. E. \& Smith, J. A. Grading of corneal and conjunctival staining in the context of other dry eye tests. Cornea 22, 640-650 (2003).

47. Efron, N. Grading scales for contact lens complications. Ophthalmic Physiol Opt 18, 182-186 (1998)

48. Dogru, M. et al. Changing trends in the definition and diagnosis of dry eyes. Am I Ophthalmol 140, 507-508 (2005).

49. Wang, Y. et al. Baseline profiles of ocular surface and tear dynamics after allogeneic hematopoietic stem cell transplantation in patients with or without chronic GVHD-related dry eye. Bone Marrow Transplant 45, 1077-1083 (2010).

\section{Acknowledgments}

We thank Mizuka Kamoi, Miki Uchino, Yukako Tatematsu, Yumiko Ban, Saori Yaguchi, Isami Hayashi and Catherine Oshima from Department of Ophthalmology, Keio University, Japan; Samantha Herretes from Bascom Palmer Eye Institute, Miami for supporting this collaborative study. This work was supported by the grant for Global 
Clinical Research Center Program for Keio University from Japanese Ministry of Health, Labor, and Welfare 2011.

\section{Author contributions}

Y.O., S.K., R.D., H.S., and K.T. wrote the main manuscript text and Y.O. prepared tables 13, and figures 1-4. All authors (Y. O., S. K., R. D., J. C., S. J., M. R., V. P., H. S., A. R., and K. T.) participate the consensus meetings, review the previous literatures and gathered the data. All authors reviewed the manuscript.

\section{Additional information}

Competing financial interests: The authors declare no competing financial interests. How to cite this article: Ogawa, Y. et al. International Chronic Ocular Graft-vs-Host-Disease (GVHD) Consensus Group: Proposed Diagnostic Criteria for Chronic GVHD (Part I). Sci. Rep. 3, 3419; DOI:10.1038/srep03419 (2013).

(c) (1) (3) This work is licensed under a Creative Commons AttributionBy Na No NonCommercial-NoDerivs 3.0 Unported license. To view a copy of this license, visit http://creativecommons.org/licenses/by-nc-nd/3.0 